\title{
Digitizing Poly-L-lysine Dendrigrafts: from Experimental Data to Molecular Dynamics Simulations
}

Jean-Patrick Francoia, ${ }^{\dagger}$ Jean-Christophe Rossi, ${ }^{\ddagger}$ Gerald Monard, ${ }^{*}, \boldsymbol{\Phi}, \S$ and Laurent

$$
\text { Vial*,ł,\| }
$$

$\dagger$ †nstitut des Biomolécules Max Mousseron, UMR 5247 CNRS - Université de Montpellier

- ENSCM, Place Eugéne Bataillon, 34296 Montpellier cedex 5, France

$\ddagger$ Institut des Biomolécules Max Mousseron, UMR 5247 CNRS - Université de Montpellier

- ENSCM, Place Eugène Bataillon, 34296 Montpellier cedex 5, France

ๆ Université de Lorraine, UMR 7565 SRSMC, Boulevard des Aiguillettes B.P. 70239,

$$
\text { F-54506 Vandoeuvre-les-Nancy, France }
$$

§CNRS, UMR 7565 SRSMC, Boulevard des Aiguillettes B.P. 70239, F-54506

Vandoeuvre-les-Nancy, France

|| Institut de Chimie et Biochimie Moléculaires et Supramoléculaires, UMR 5246 CNRS -

Université Claude Bernard Lyon 1 - CPE Lyon - INSA, 43 Boulevard du 11 Novembre 1918, 69622 Villeurbanne cedex, France

E-mail: gerald.monard@univ-lorraine.fr; laurent.vial@univ-lyon1.fr

\begin{abstract}
Despite of a growing use of the poly- $L$-lysine dendrigrafts in biomedical applications, a deeper understanding of the molecular level properties of these macromolecules
\end{abstract}


is missing. Herein, we report a simple methodology for the construction of threedimensional structures of poly- $L$-lysine dendrigrafts, and the subsequent investigation of their structural features using microsecond molecular dynamics simulations. This methodology relies on the encoding of the polymers- experimental characterizations (i.e. composition, degrees of polymerization, branching ratios, charges) into alphanumeric strings that are readable by the Amber simulation package. Such an original approach opens avenues toward the in silico exploration of dendrigrafts and hyperbranched polymers.

\section{Introduction}

Dendrigrafts of poly-L-lysine (DGLs) recently complemented the family of polycationic dendritic macromolecules that include the prominent polyamidoamine (PAMAM) and polyethylenimine (PEI) polymers (Figure 1). ${ }^{1}$ Because their iterative synthesis in aqueous conditions is thermodynamically controlled by precipitation and kinetically controlled by steric hindrance, DGLs share features with both PAMAM dendrimers (i.e. generation-based growth and narrow molecular weight distribution), and hyperbranched PEI (i.e. broad number of regioisomers and rapid increase in molecular weight per generation). In contrast to PAMAM and PEI, DGLs are biodegradable, ${ }^{2}$ exhibit low cellular toxicities, ${ }^{3}$ and turned out to be non-immunogenic. ${ }^{4}$ As a consequence, DGLs have recently gained a huge interest in the biomedical field during the last quinquennium, with numerous applications in drug and gene

delivery, ${ }^{2,3,5-18}$ biomaterials and tissue engineering, ${ }^{19-25}$ bio-imaging ${ }^{26-31}$ and biosensing. ${ }^{32-34}$ Despite this growing use of DGLs, a deeper understanding of the molecular level properties of these macromolecules is missing. In this context, we decided to explore the possibility to construct DGLs in silico and subsequently perform molecular dynamics (MD) simulations. 


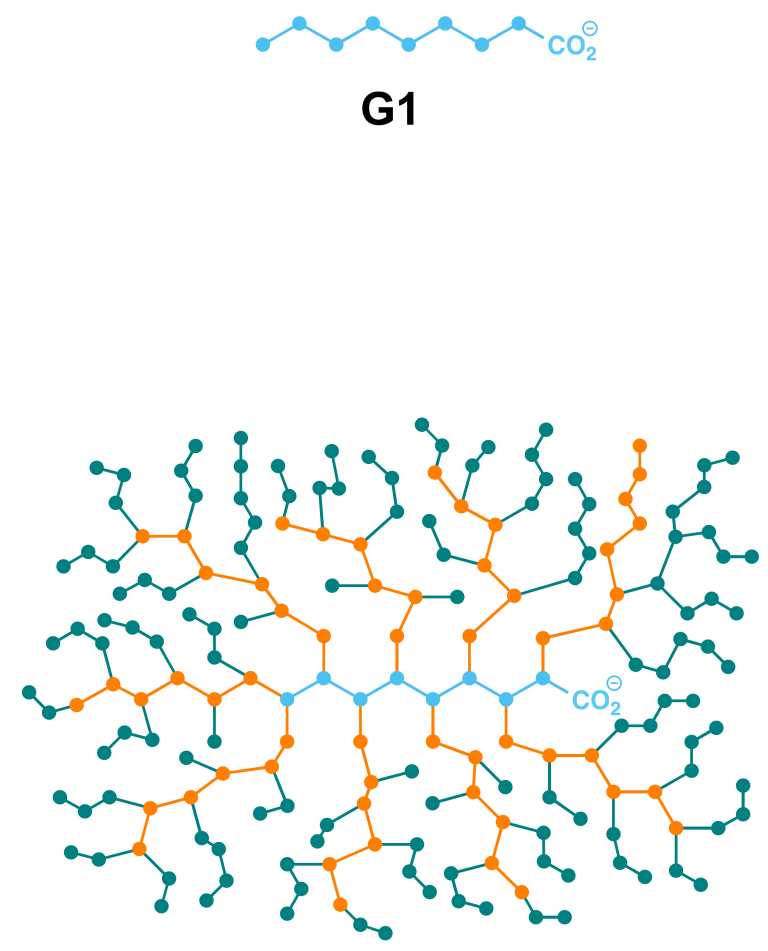

G3

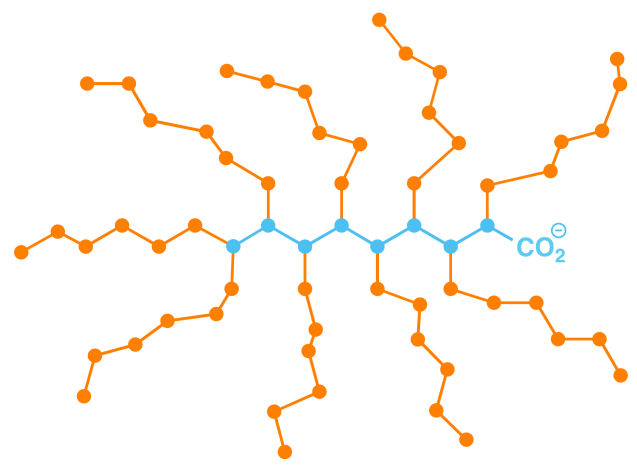

G2

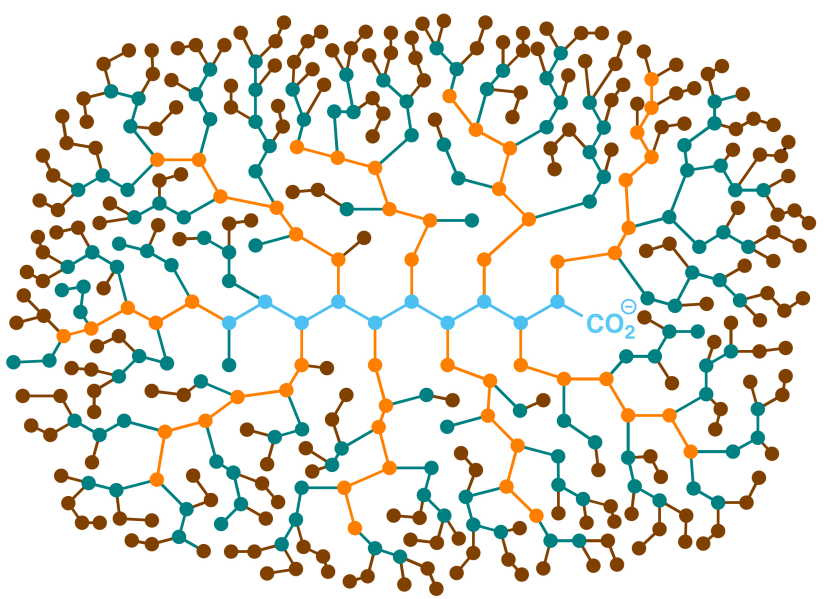

G4

Figure 1: Schematic representation of the first- to fourth-generation DGLs G1-G4 (each dot represents a $L$-lysine residue, pending free amino groups are not represented). 


\section{Results and Discussion}

\section{Experimental Data}

As DGLs are only made of $L$-lysine amino acids, one could assimilate them to proteins. However, some of the residues are linked to each other through isopeptidic bonds (i.e. amide linkages that involve the nitrogen atom of the lateral chain of lysine residues). From the data that were previousy collected in our group, ${ }^{1}$ we extracted the degree of polymerization obtained from size exclusion chromatography (DP) and the mean branching ratio obtained from ${ }^{1} \mathrm{H}$ NMR spectroscopy (BR, defined as the ratio of the number of $\epsilon$-branched Lysine residues over the total number of residues DP), which allowed us to calculate the number of peptidic and isopeptidic bonds, as well as the number of free $\alpha$ - and free $\epsilon$-amine functions for each dendrigraft generation G1-G4 (Table 1). Also, determining the protonation state at physiological $\mathrm{pH}$ of each generation of DGL was obviously required in order to perform accurate modeling experiments. When a molecule bears several acidic functions, the protonation state of each of them influences the intrinsic acidity of the other ones, resulting in a shift of the functions' pKa. This phenomenon is called the polyelectrolyte effect. ${ }^{35}$ Moreover, these pKa are also influenced by several other parameters, such as the solvent exposition of the acidic functions, or their capacity to form hydrogen bonds. While the pKa of the $\alpha$-amine in an isolated lysine residue is 9.16 , the literature reports an average value of 7.7 for proteins' N-termini, with a low value of $6.8 .{ }^{36,37}$ We performed $\mathrm{pH}$-metric titrations on DGLs (with trifluoroacetate TFA as counterions) in deionized water. The samples were fully acidified with $0.5 \mathrm{~mL}$ of $1 \mathrm{M}$ nitric acid, and then submitted to incremental additions of 0.5M sodium hydroxide using a $702 \mathrm{SM}$ Titrino automatic titrator. The resulting titration curves are shown in figure 2. 

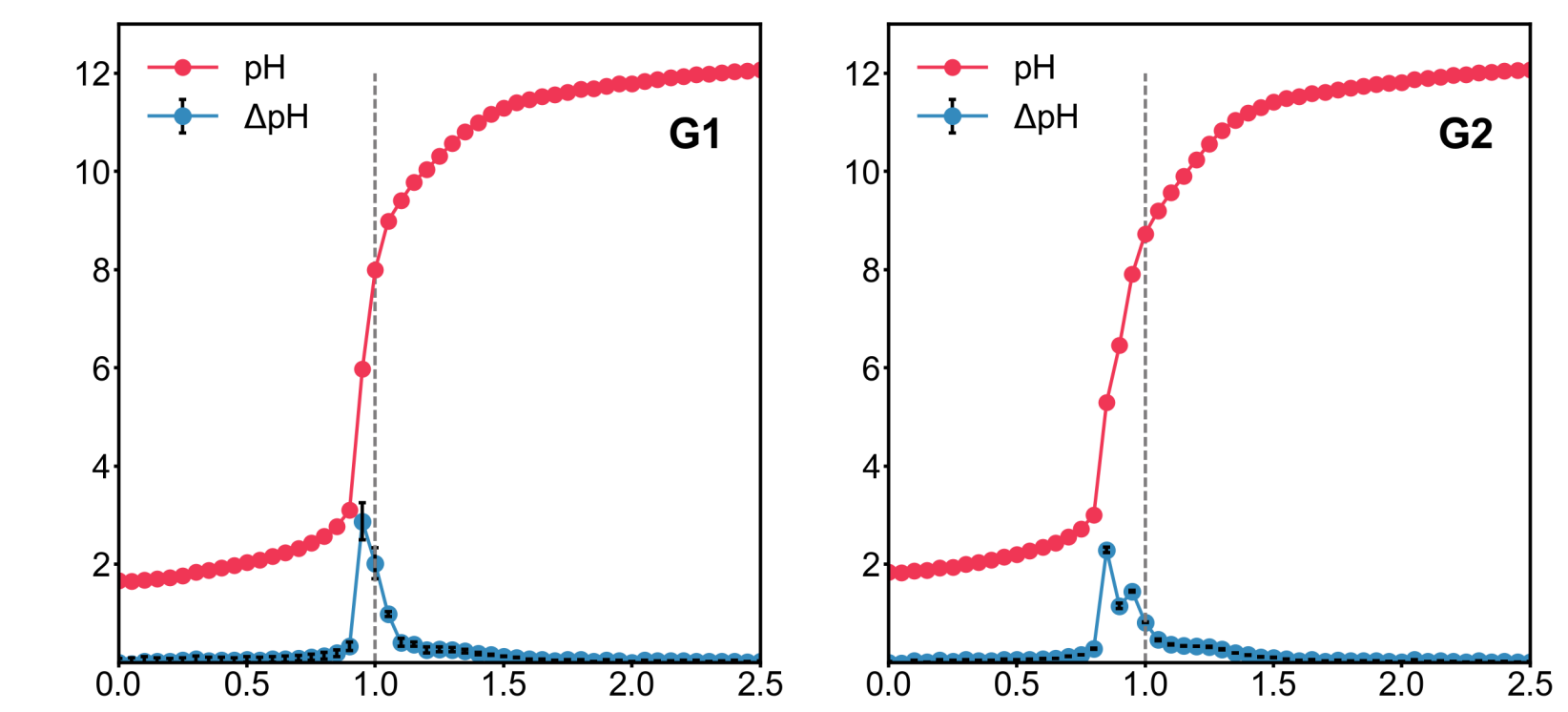

I
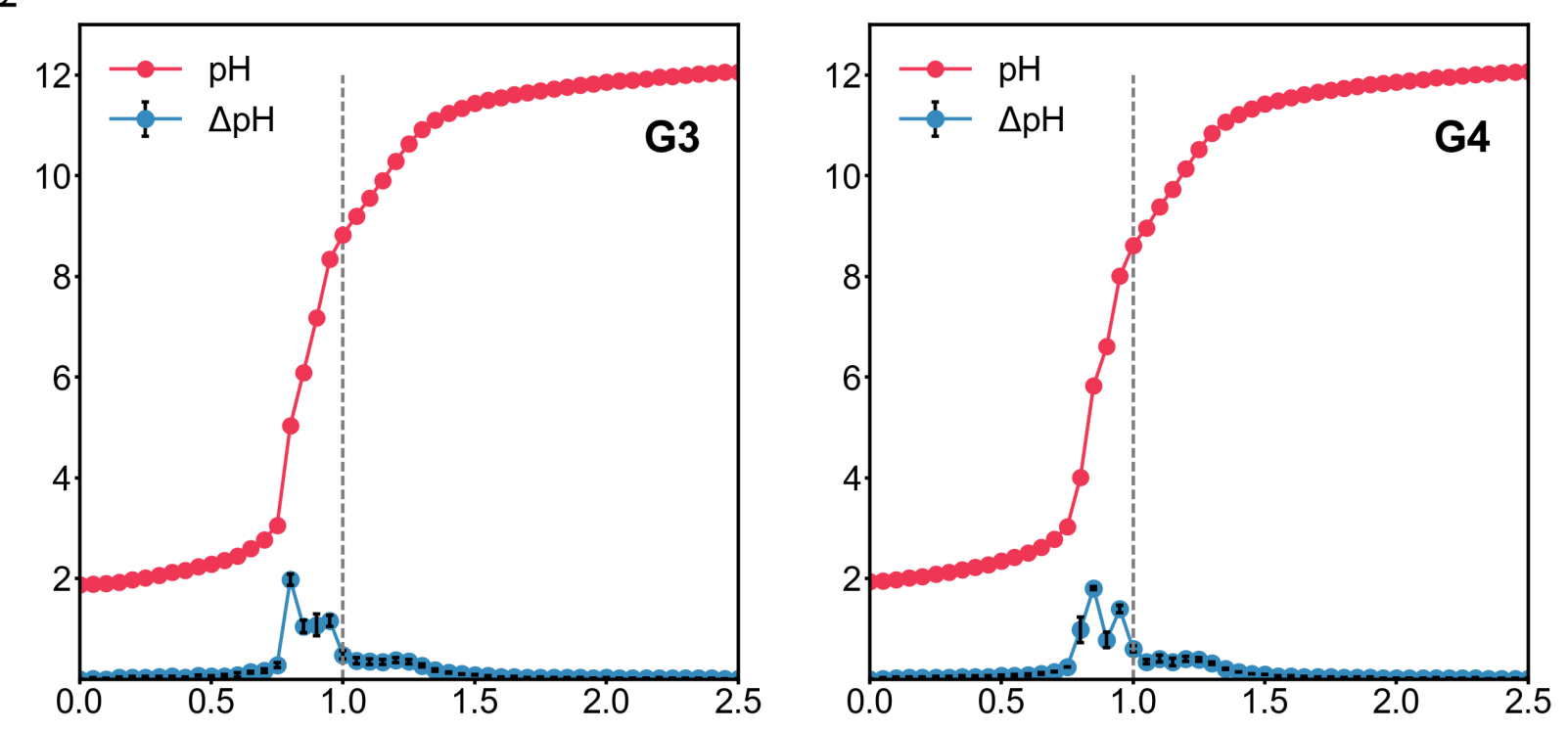

Volume of $\mathrm{NaOH}(\mathrm{mL})$

Figure 2: pH-metric titrations of DGLs G1-G4 in deionized water. Error bars are calculated from the standard deviation of each $\mathrm{pH}$ measurement.

One would expect the $\mathrm{pH}$ to be stable below $1 \mathrm{~mL}$ of added $\mathrm{NaOH}$ since it was the required volume to neutralize the amount of nitric acid used to acidify the samples. However all the titration curves displayed a "lack" of nitric acid. This gap could be explained by the fact that not all the amine functions were protonated in the commercial powders. ${ }^{38}$ Thus, we made the hypothesis that a part of the nitric acid added in a first place was consumed by 
the protonation of these neutral amino groups. The second peaks of the derivatives allowed us to calculate the number of $\alpha$-amino groups in the samples, except for DGLs G1 since the presence of a unique $\alpha$-amino group did not result in a mesurable signal by potentiometry. Due to the polyelectrolyte effect, the third peaks of the derivatives - corresponding to the pKa of the $\epsilon$-amines - were barely visible. The deprotonation of these functions takes place on a wide range of $\mathrm{pH}$, making it impossible to determine the number of $\epsilon$-amines, which was therefore calculated from the ratio $\frac{\alpha}{\epsilon}$ (see table 1 ). The electroneutrality equation was then used to calculate the protonation fraction $\Theta$ (equation 1 ), where $C_{a}$ is the concentration of added acid, $C_{b}$ the concentration of added base, $C_{T F A}$ the missing concentration of TFA, $C_{p}$ the total concentration of amino groups, and $C_{H^{+}}$and $C_{\mathrm{OH}^{-}}$the concentration of free $\mathrm{H}^{+}$ and free $\mathrm{OH}^{-}$, respectively.

$$
\begin{gathered}
\Theta=\frac{C_{a}-C_{b}-C_{T F A}-C_{H^{+}}+C_{O H^{-}}}{C_{p}} \\
\Theta=\frac{C_{a}-C_{b}-C_{T F A}-10^{-p H}+\frac{10^{-p K_{w}}}{10^{-p H}}}{C_{p}}
\end{gathered}
$$

Equation 1 could also be expressed as a function of the $\mathrm{pH}$, leading to equation 2. Applied on the experimental titration data, equation 2 gave the protonation degree of DGLs G1-G4 at a physological pH of 7.8, which was selected since all the reported applications for DGLs are in the biomedical field (Table 2). It turned out that this percentage of non-protonated amine functions are in excellent agreement with the percentage of $\alpha$-amine functions in the DGLs. This suggested that only the $\epsilon$-amines were protonated at $\mathrm{pH} 7.8$. 
Table 1: Selected properties of DGLs G1-G4. DP: degree of polymerization; BR: branching ratio.

\begin{tabular}{ccccccc}
\hline DGL & DP & $\begin{array}{c}\text { BR } \\
(\%)\end{array}$ & $\begin{array}{c}\text { Free } \alpha \text {-amino } \\
\text { groups }\end{array}$ & $\begin{array}{c}\text { Free } \epsilon \text {-amino } \\
\text { groups }\end{array}$ & Isopeptidic bonds & Peptidic bonds \\
\hline G1 & 8 & 0 & 1 & 8 & 0 & 7 \\
G2 & 48 & 12 & 7 & 42 & 6 & 41 \\
G3 & 123 & 24 & 31 & 93 & 30 & 92 \\
G4 & 365 & 23 & 85 & 281 & 84 & 280 \\
\hline
\end{tabular}

Table 2: Protonation state of DGLs G1-G4 at $\mathrm{pH}$ 7.8. $\Theta$ : protonation fraction; $\epsilon$ : number of free $\epsilon$-amino groups; $\alpha$ : number of free $\alpha$-amino groups. We assumed that, as for DGLs G2-G4, only the $\epsilon$-amino groups of DGL G1 were protonated.

\begin{tabular}{cccc}
\hline DGL $\Theta(\%)$ & $\frac{\epsilon}{\epsilon+\alpha}(\%)$ & Net charge \\
\hline G1 & N/A & N/A & +7 \\
G2 & 86 & 86 & +41 \\
G3 & 79 & 75 & +92 \\
G4 & 79 & 78 & +280 \\
\hline
\end{tabular}




\section{Encoding}

From these experimental parameters, a manual approach toward an initial set of threedimensional (3D) structures for DGLs may suffer from several flaws: i) it is highly timeconsuming to manually edit starting structures that contain all atoms and residues with a correct naming in a coherent order, as well as no crossing nor bumping between the branches, ii) it is very much error-prone since the fourth-generation $\mathbf{G} 4$ consists of 365 residues, iii) it is likely to be biased toward an homogeneous distribution of the residues and unlikely to be representative of the experimental characteristics of the polymers. For these reasons, a new and automated method has been designed for the construction of 3D structures of DGLs. As a first step, the macromolecules G1-G4 have been deconvoluted into six discrete building blocks, which encompass all the possible arrangements (i.e. peptidic or isopeptidic bonds, charged or neutral nitrogen atoms) of the $L$-lysine residues within the dendrigrafts (Figure 3). Each building block was subsequently labeled with a one-letter code that unambiguously identifies it. DGL G1 could be written as the following alphanumeric string: ACCCCCCh (from C-terminal to N-terminal). It should be highlighted that DGLs were analogously synthetized in silico and at the bench, with the growth of the chains from C-terminal to the $\mathrm{N}$-terminal using $\mathrm{N} \epsilon$-protected- $L$-lysine residues. Moving to a higher generation required the deprotection of the lysines- side chains, and elongation/branching/termination events from the free nitrogen atoms. ${ }^{1}$ For the higher generation $\mathbf{G 2 - G 4}$, numbers have been introduced within the strings, which addressed the locations of the residues where branched chains were growing from (starting from the C-terminal amino acid). For instance, a possible representation of a G2 polymer using these molecular descriptors would be an alphanumeric string of 48 letters, and 6 discrete numbers (Figure 4, Top). As a result, these alphanumeric strings encoded not only the position of every $L$-Lysine residues in the macromolecules, but also their connections and protonation states (Figure 4, Bottom). The algorithm that built this sequence works as follows (Figure 5). Starting from a single monomer, the chain was elongated through the iterative formation of peptidic bonds until the DP (=8) of DGL G1 was reached. 
Then, $6(=\mathrm{DP} \times \mathrm{BR})$ residues were randomly branched to $\mathbf{G} \mathbf{1}$ in order to respect the experimental number of isopeptidic bonds. A loop was subsequently used to randomly grow the polymer through peptidic bonds until the DP $(=48)$ of DGL G2 was reached. Finally, the algorithm walked through the tree and translated it into the following alphanumeric string BCDCDDDDCCh8CCCCCh7CCCCCCCCCh6CCCCCh5CCCCh3CCCCh1CCCCh, which encodes one possible structure of DGL G2. Using this algorithm, a homemade python script $^{39}$ allowed us to generate eight random - but respecting the initial experimental parameters (i.e. DP, BR, and net charge) - sequences for each generation of DGL G2 to G4 (see ESI $\dagger$ ). Within a set, each new generation of DGL was grown from the corresponding lower one.

The coded polymers could then be converted to candidate 3D structures by using a customized Nucleic Acid Builder (NAB) program ${ }^{40}$ from the Amber simulation package. ${ }^{39,41}$ To put it simply, each one-letter coded residue was sequentially read by the program (from C-terminal to N-terminal), translated to a three-letter equivalent in order to satisfy the PDB three-letter conventional naming of the residues required by AMBER preparatory tools (see Figure 3), converted to its corresponding 3D molecular building block, and finally added at the right position to the growing dendrigraft. Preparatory ab initio quantum mechanics calculations (Hartree-Fock method, at the 6-31G level) were performed in order to assign atomic partial charges for the six building blocks A-h by using the Antechamber module of the Amber simulation Package. After each addition, the positions of all current atoms were optimized using a short conjugated gradient minimization (cut-off of $15 \AA$, with a maximum of 100 iterations) in order to ensure that no bond or atom overlapping was caused.

\section{Molecular Dynamics}

We performed an investigation on the structural features of DGLs by using MD simulations on the full set of 3D structures of the dendrigrafts that were generated previously (1 structure for DGL G1, 8 structures for each upper generation, 25 polymers in total). All-atom 

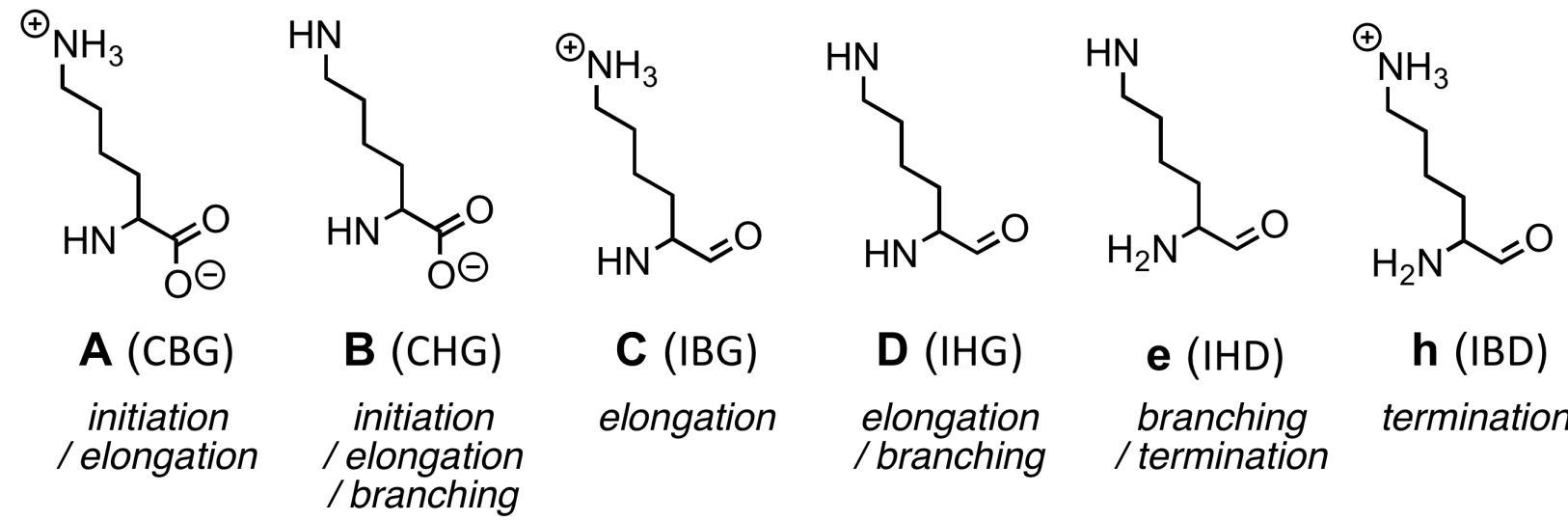

D (IHG)

e (IHD)

h (IBD)

elongation

elongation

/branching

branching
/ termination

termination

Figure 3: All possible $L$-Lysine buildings blocks $\mathbf{A}-\mathbf{h}$ (and their corresponding three-letter codes in brackets) involved in the construction of DGLs G1-G4.
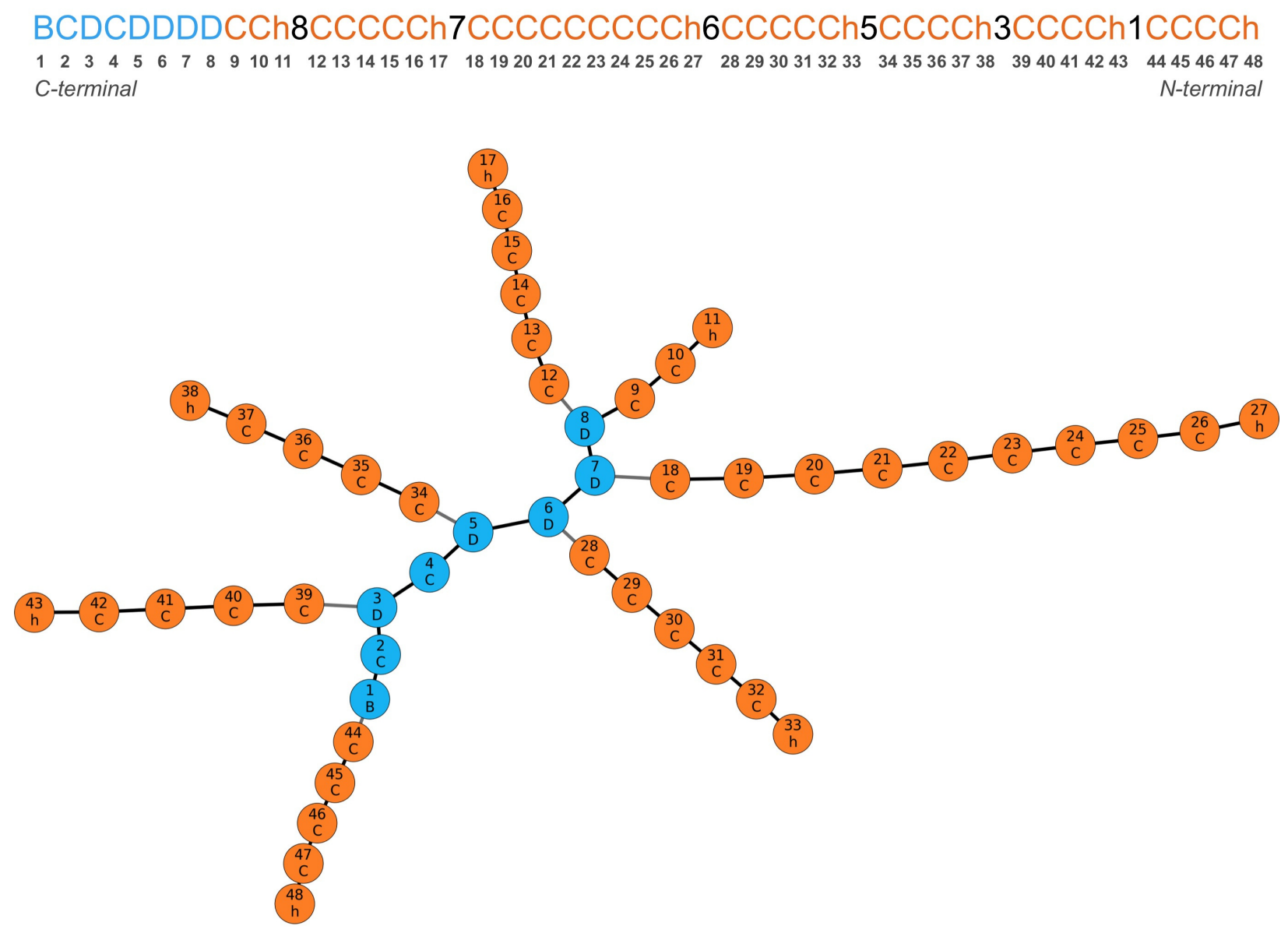

Figure 4: The alphanumeric description of a second-generation dendrigraft G2 (Top), and the corresponding arrangement of the residues (Bottom). Residues in blue indicate the mother DGL G1. Peptidic and isopeptidic bonds are depicted as black line and grey lines, respectively. 


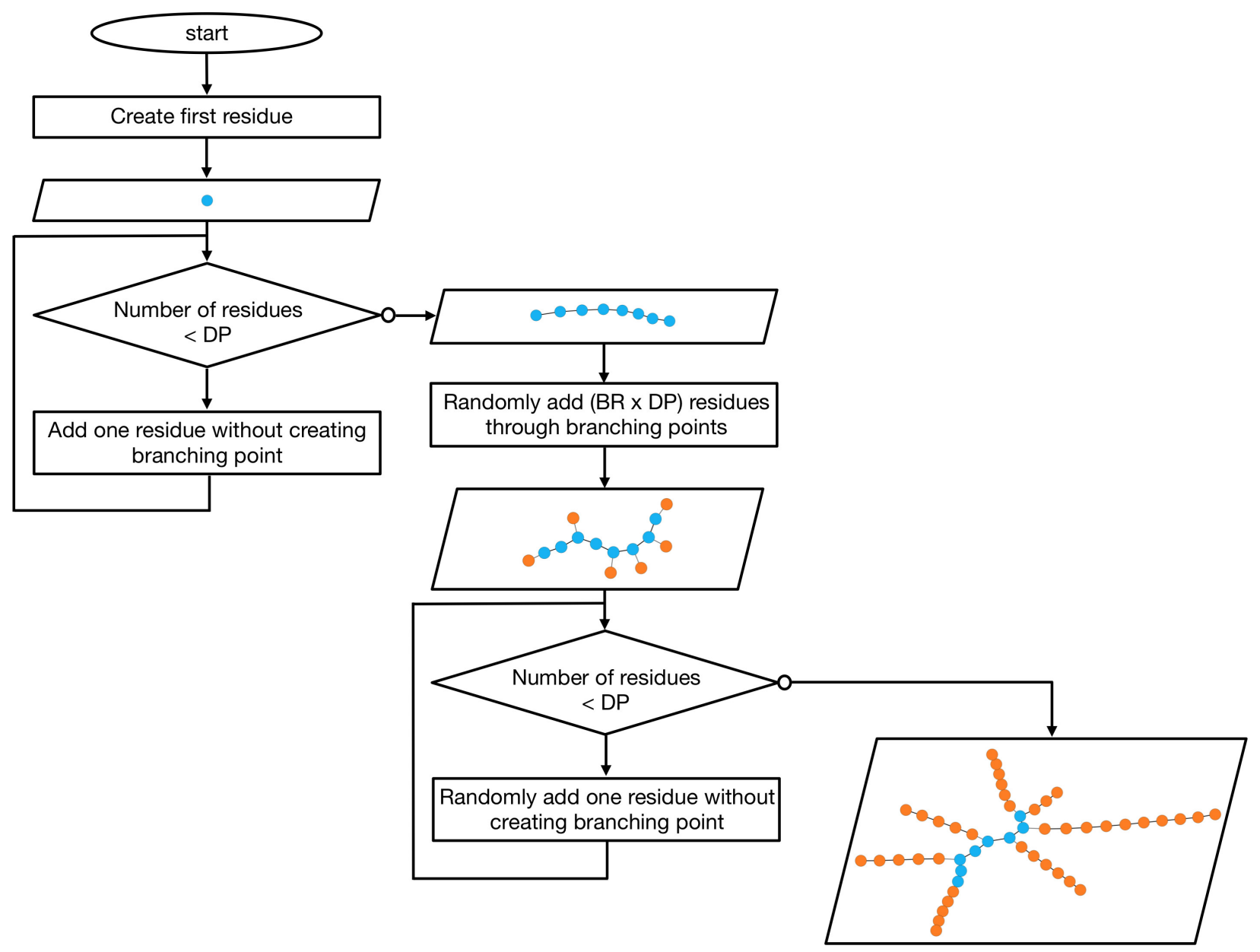

Figure 5: Flowchart toward the encoding of one possible strucure of DGL G2, which respects its experimental characteristics. 
classical MD simulations were run on NVIDIA graphics processing units and included the generalized Born solvation model, which previously proved to yield the best agreement between calculated and experimental structures for proteins. ${ }^{42}$ Also, the method of hydrogen mass repartitioning was applied for further accelerating the simulations. ${ }^{43}$ Using this setup, trajectories of 10 microseconds for first- and second-generation DGLs, and of 1 microsecond for third- and fourth-generation DGLs could be obtained within reasonable calculation time scales. The mean calculation times (with simulation times in parentheses) on NVIDIA K20 GPUs were $207.6 \mathrm{~h}(10 \mu \mathrm{s})$ for G1, 362.6 h $(10 \mu \mathrm{s})$ for G2, $78.4 \mathrm{~h}(1 \mu \mathrm{s})$ for G3, and 432.2 h $(1 \mu \mathrm{s})$ for G4. At this point, it should be noted that - albeit each dendrigraft within a same generation displays a unique topology - the analyses of the trajectories did not revealed divergent dynamic behaviours. As a consequence, the post-calculation analyses for only one dendrigraft per generation are shown in the manuscript (for the full set, see ESI $\dagger$ ).

Via the Flory theory approach, we obtained the scaling relation $R g \sim N^{0.41}$, where $R g$ is the radius of gyration and $N$ the number of residues of the dendrigrafts. ${ }^{44}$ The exponent of the power law lies in between the calculated values at physiological $\mathrm{pH}$ of 0.34 and 0.57 for poly- $L$-lysine dendrimers and linear poly- $L$-lysines, respectively. ${ }^{45-47}$ This suggests that the dendrigrafts adopt intermediate morphologies, which may remind those of unfolded and intrinsically disordered proteins. ${ }^{48}$ This conformational flexibility was further supported by the $2 \mathrm{D}$ root-mean-square deviations of selected residues ${ }^{49}$ (see ESI $\dagger$ ) and the Ramachandran plots of all possible residues (data not shown) along the MD trajectories, from which it was clear that no folded structure was clearly identifiable for any of the 4 generations of dendrigraft G1-G4.

In order to assess the shape of the dendrigrafts, the asphericity parameter $(\Delta)$ and shape parameter $(S)$ can be evaluated from the inertial tensor ${ }^{50}$ described in equation 3 , where $M$ is the total mass, $m_{i}$ and $r_{i}$ are the mass and position of the $\mathrm{i}^{\text {th }}$ residue, and $\alpha, \beta=x, y, z$.

$$
T_{\alpha, \beta}=\frac{1}{2 M^{2}} \sum^{N} \sum_{j}^{N} m_{i} m_{j}\left(r_{i \alpha}-r_{j \alpha}\right)\left(r_{i \beta}-r_{j \beta}\right)
$$


The squares of the three principal radii of gyration- $R_{G^{-}}$are calculated from the trace of the matrix $-T(\alpha, \beta)$, which amounts to the sum of its eigenvalues- $\lambda_{1},-\lambda_{2}$ and $-\lambda_{3}$ (equation $4)$.

$$
R_{G}^{2}=\operatorname{tr} T=\sum_{i=1}^{3} \lambda_{i}
$$

These eigenvalues are used to calculate $\Delta$, which measures the average deviation from spherical symmetry (equation 5).

$$
\Delta=\frac{3}{2} \frac{\sum_{i=1}^{3}\left(\lambda_{i}-\lambda\right)^{2}}{(t r T)^{2}}
$$

Where

$$
\lambda=\frac{\sum_{i=1}^{3} \lambda_{i}}{3}
$$

On the other hand, $S$ further describes the ellipticities of the polymers. In three dimensions, $S$ can be calculated from equation 7 .

$$
S=27 \frac{\prod_{i=1}^{3}\left(\lambda_{i}-\lambda\right)}{(\operatorname{tr} T)^{3}}
$$

When applied to our trajectories, these treatments gave almost null $\Delta$ and $S$ values (see $\mathrm{ESI} \dagger$ for full set of values). While the theoretical lowest value of $\Delta$ is zero (i.e. characterizing a sphere) and the highest value is one (i.e. characterizing a rod), the values of $S$ are theoretically bounded following the inequality $-\frac{1}{4} \leq \mathrm{S} \leq 2$, with negative and positive values indicating oblate and prolate objects, respectively. When applied to our trajectories, the calculated values actually establish that our dendrigrafts are mostly globular, as they previously were represented in the literature (Table 3), with the exception of the first-generation G1 that slightly tends toward a disc-shaped rod due to its linear topology. Insights into the spatial occupancy of the macromolecules were gained by combining the atomic coordinates 
of each frame - captured every nanosecond for G1 and G2, and every 100 picoseconds for G3 and G4 - over the entire MD trajectory. The resulting volumetric maps showed that the DGLs tend to occupy a large amount of the available space as a result of the flexibility of their branches (Figure 6).

Table 3: Averages and standard deviations of the asphericity $(\Delta)$ and shape $(S)$ parameters for first- to fourth-generation DGLs G1 to G4.

\begin{tabular}{ccccc} 
& G1 & G2 & G3 & G4 \\
\hline$\Delta$ & $0.107 \pm 0.034$ & $0.029 \pm 0.014$ & $0.023 \pm 0.007$ & $0.008 \pm 0.002$ \\
$S$ & $-0.069 \pm 0.032$ & $-0.003 \pm 0.006$ & $-0.004 \pm 0.003$ & $-0.001 \pm 0.001$ \\
\hline
\end{tabular}

Then, we plotted both the normalized atomic and cationic densities as a function of their distances from the center of mass of the polymers (Figure 7). ${ }^{51}$ Such an analysis revealed that: i) the atomic density shifts from the core toward the outside of the dendrigrafts with increasing generation, and ii) the cationic charges are mainly found on the outer shell layer of the dendrigrafts. The distribution of the positive charges, as well as the bulk density of the dendrigrafts, can also be appreciated from snapshots taken after $1 \mu \mathrm{s}$ of the trajectories (Figure 8). Finally, we measured the distance between all adjacent charged nitrogen atoms (NZ) using the pair correlation function (Figure 9). It turned out that all DGLs independently to their generation - displayed adjacent NZ separated by a similar average distance of $8.0 \AA$ (first peak of the curves). This latter observation relates to the synthetic route towards DGLs, ${ }^{1}$ which implies a $\mathrm{N}^{\epsilon}$-protected lysine derivative and therefore excludes an impact of the electrostatic repulsion on the ionizable nitrogen atoms' distribution. 


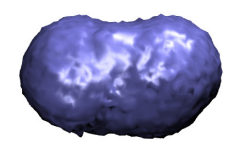

G1

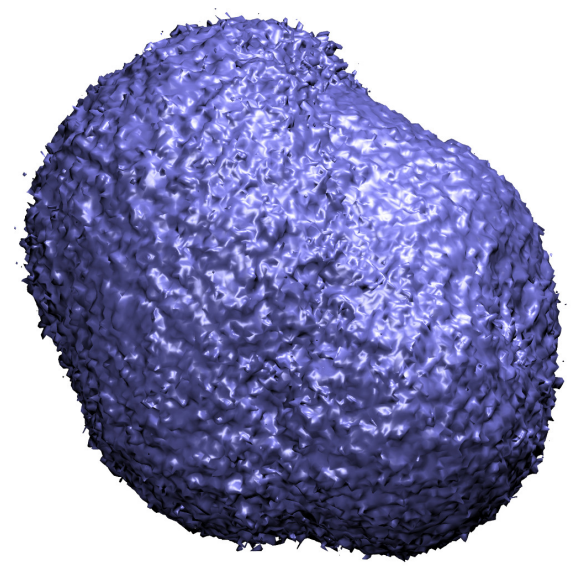

G3

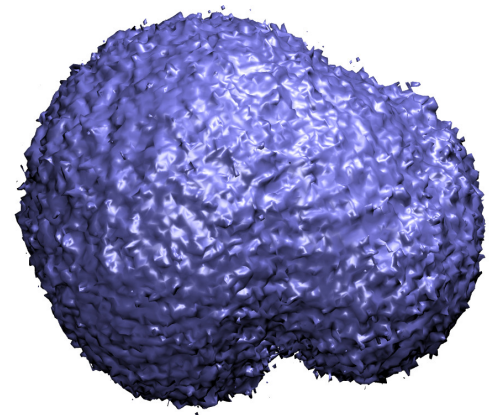

G2

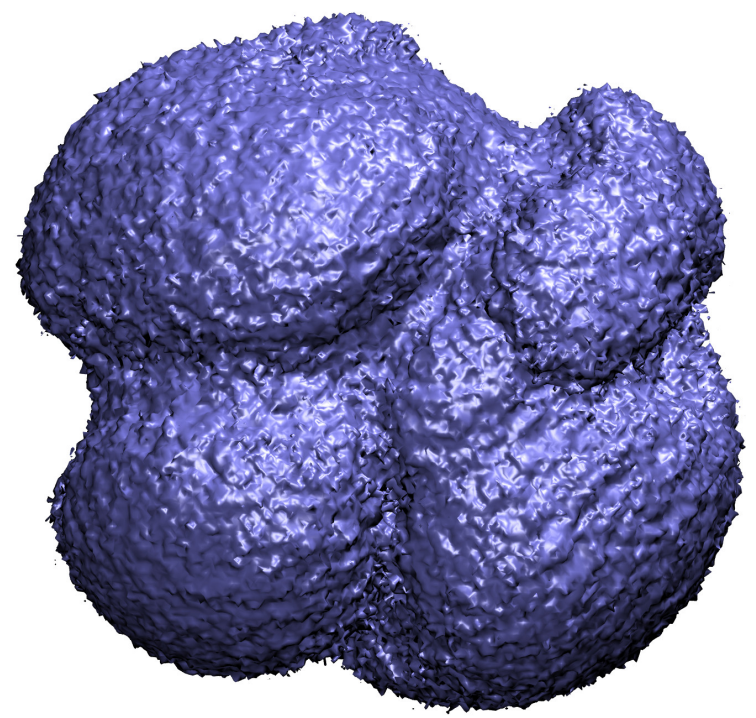

G4

Figure 6: Volumetric maps of first- to fourth-generation DGLs G1-G4 at 1 microsecond with $99 \%$ probability boundary surfaces. All images are to the same scale. Images were generated with VMD (ref. 52). 


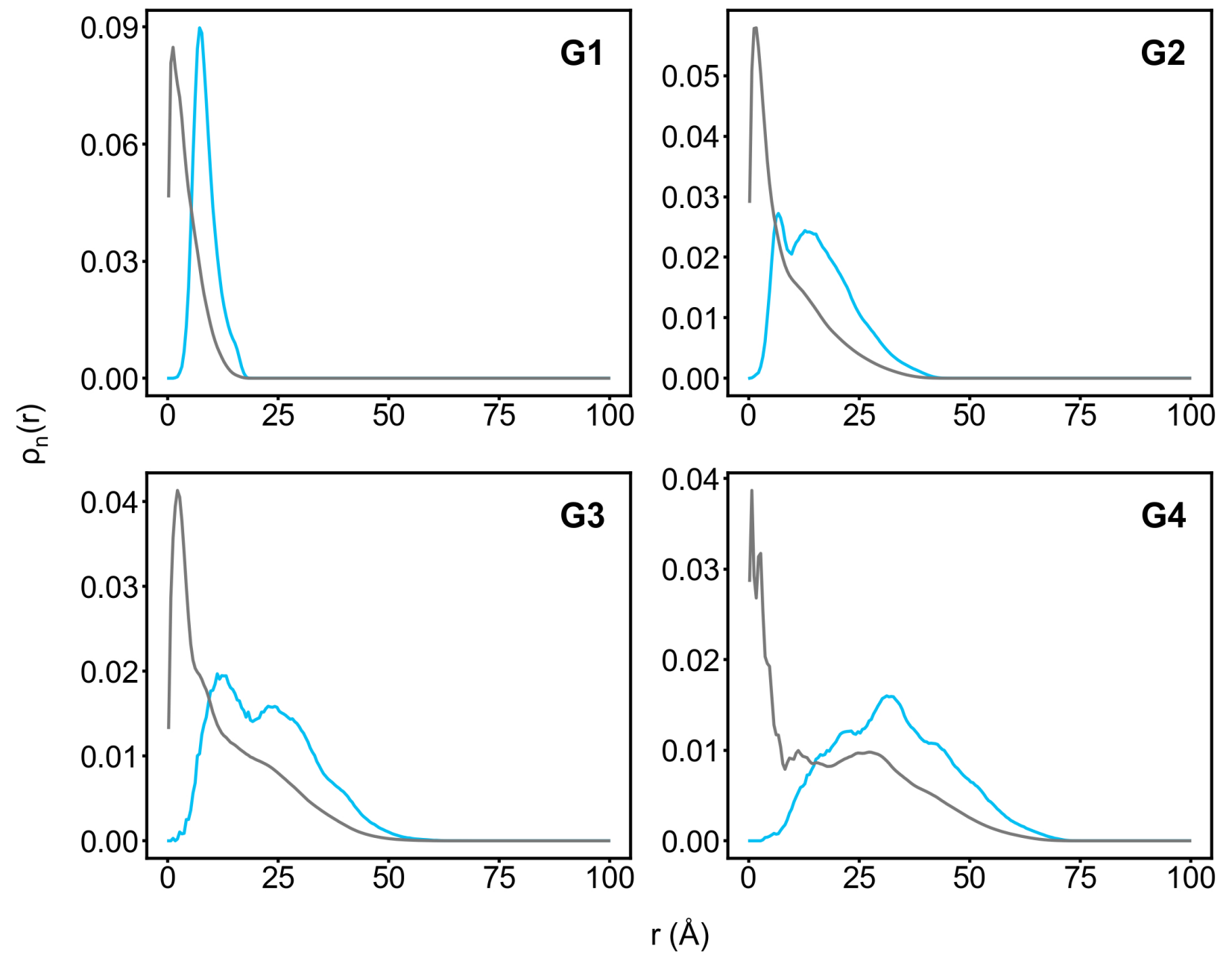

Figure 7: Normalized atomic (in grey) and cationic (in blue) density profiles of first- to fourth-generation DGLs G1-G4 (ref. 51). 


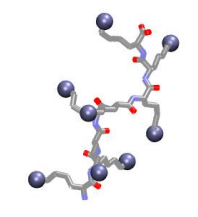

G1

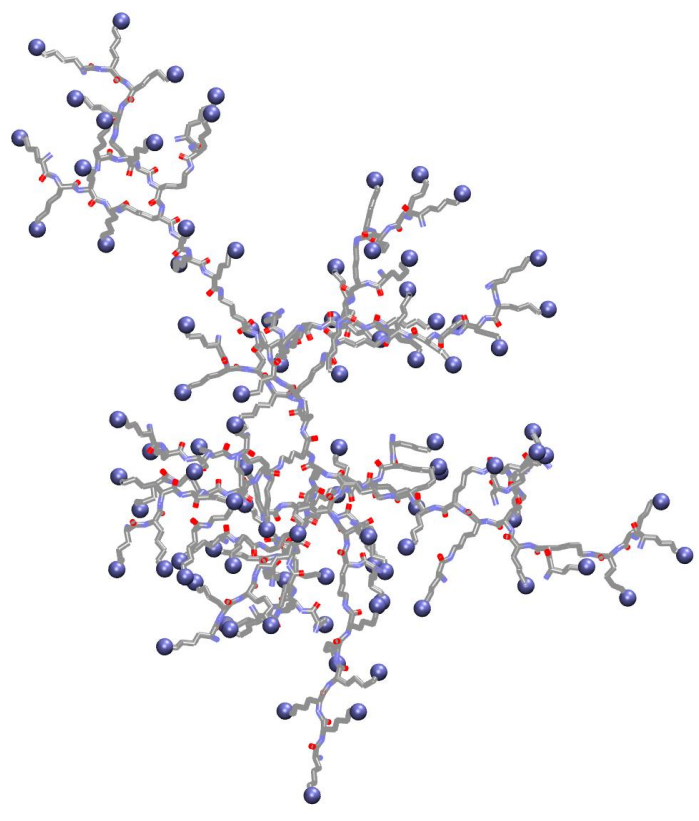

G3
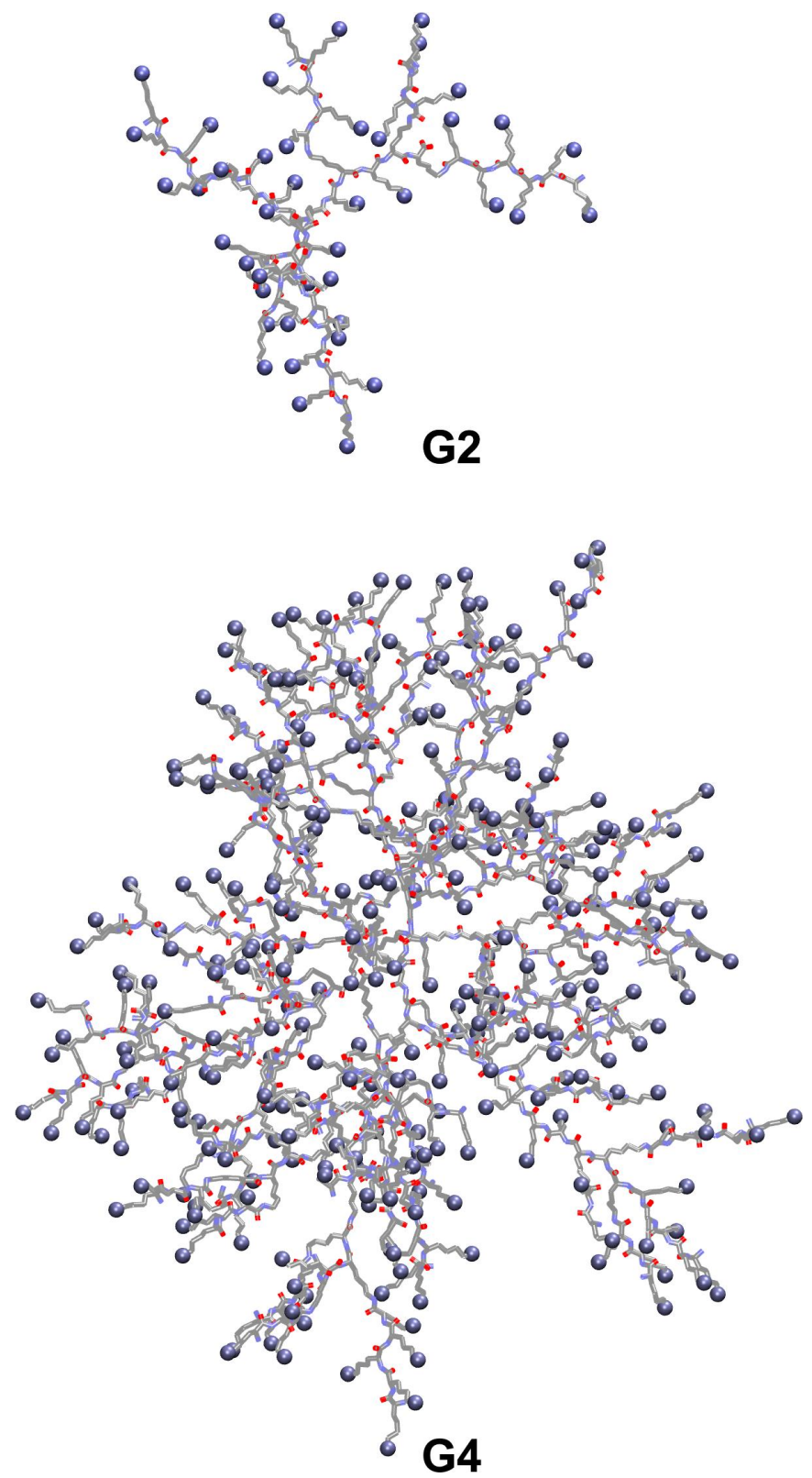

Figure 8: Snapshots of the first- to fourth-generation DGLs G1 to G4 taken after 1 microsecond at $\mathrm{T}=300 \mathrm{~K}$. Blue dots highlight the positively charged nitrogen atoms at $\mathrm{pH}$ 7.8. All images are to the same scale. 


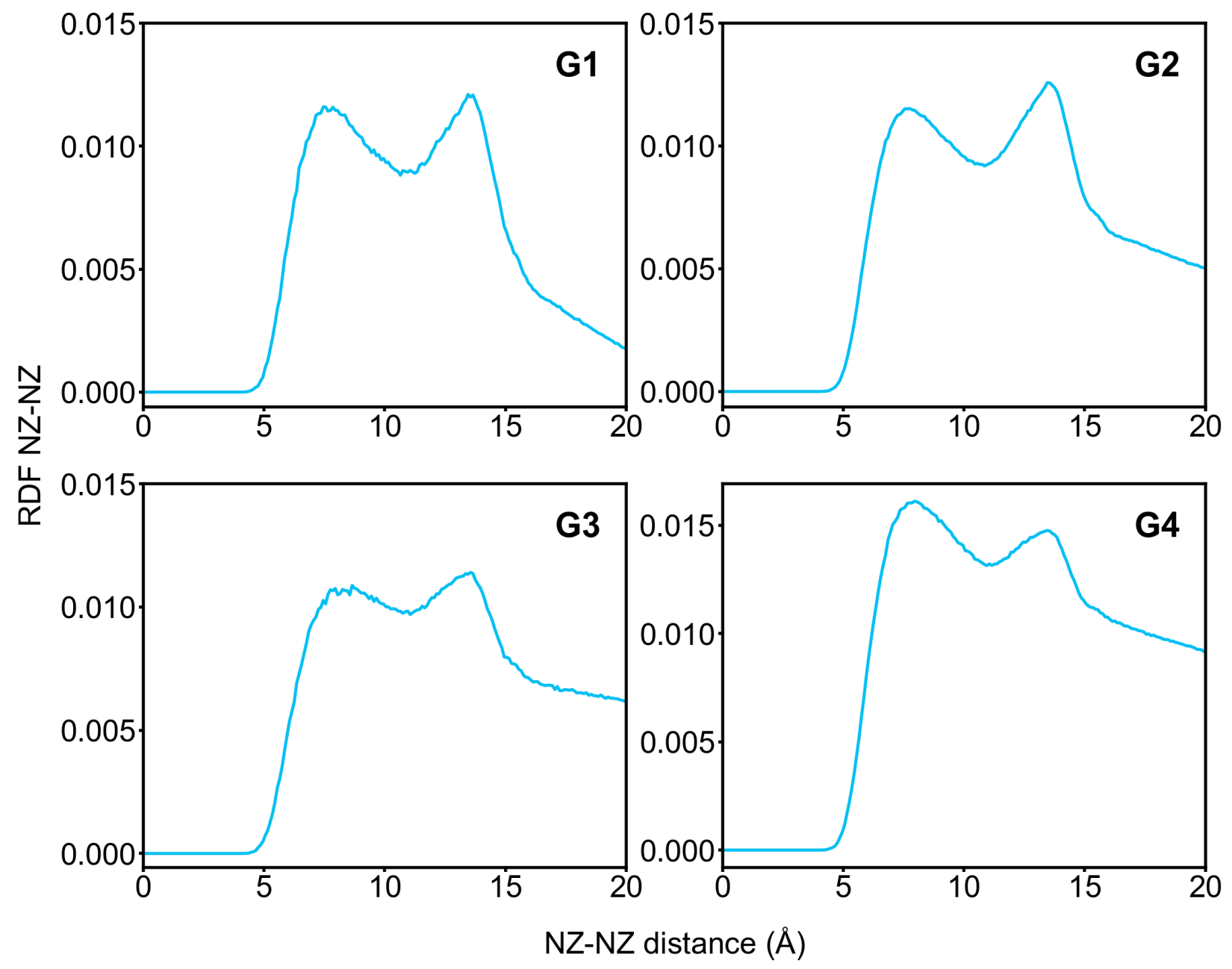

Figure 9: Radial Distribution Function (RDF) of the charged nitrogen atoms (NZ) for the first- to fourth-generation $\mathbf{G} \mathbf{1}$ to $\mathbf{G} 4$.

By being able to generate accurate 3D structures of four generations of poly- $L$-lysines dendrigrafts with respect to their experimental characteristics (i.e. composition, degrees of polymerization, branching ratios, number of charges at physiological $\mathrm{pH}$ ), and to determine their molecular properties (i.e. topology, shape, flexibility), one should now be able to study at the molecular level their interactions with biologically relevant partners such as drugs, metabolites, biopolymers, genetic materials, proteins, or cells. These computational studies may not only provide important information to apprehend their unique features such as biocompatibility, ${ }^{3,4,12}$ membrane translocation ability, ${ }^{12}$ and highly efficient electrostatic binding property, ${ }^{32}$ but consequently also lead to the development of DGLs for real-world 
applications in nanomedicine.

\section{Computational Details}

Residues were prepared with the antechamber module of AmberTools. ${ }^{41}$ Atomic charges were generated from RESP charges extracted from $\mathrm{HF} / 6-31 \mathrm{G}^{*}$ optimized geometries of capped residues. Initial structures were built using the Nucleic Acid Builder (NAB) program ${ }^{40}$ and the LEaP module of AmberTools. From the primary sequence of one dendrimer, new residues were added one by one and, at each new addition, a minimization of the full system was performed for 100 steps. All molecular dynamics simulations were carried out using the GPU implementation ${ }^{53}$ of the pmemd program in Amber $15^{41}$ with the combination of GB-Neck2 model, ${ }^{54}$ mbondi3 radii, ${ }^{54}$ and ff14SB. ${ }^{55}$ No cutoff was applied for computing the

nonbonded interactions. A time step of 4 fs was used with hydrogen mass repartitioning. ${ }^{56}$ Bonds involving hydrogen were constrained by the SHAKE algorithm. ${ }^{57}$ Temperature was controlled with a Langevin thermostat at $300 \mathrm{~K}$ with a collision frequency $\gamma=1.0 \mathrm{ps}^{-1}$.

\section{Conclusions}

In conclusion, microsecond molecular dynamics simulations on second- to fourth-generation poly- $L$-lysine dendrigrafts revealed a myriad of conformational states accessible to them without folded states. This conformational plasticity (i.e. this ability to adaptably display positive charges to make ion pairs) may explain why DGLs are such efficient binders for anionic ligands even in the most competitive media. In addition, the generated structures can now be exploited for in-depth analyses of the interactions between these macromolecules and biologically relevant partners for future biomedical applications. More broadly, this work provides to the community a general approach toward the construction of dendrigrafts - but also hyperbranched polymers - in silico. We are therefore confident that molecular dynamics will become a pervasive tool to explore the structural features of large dendrigrafts 
and hyperbranched polymers at the molecular level.

\section{Acknowledgement}

This work was supported by the Centre National de la Recherche Scientifique (CNRS), the Université de Lorraine, and the Université de Montpellier. GPU resources were allocated by the Centre de Calcul ROMEO of the Université de Reims Champagne-Ardenne. DGLs were supplied by the COLCOM company (Montpellier, France).

\section{Supporting Information Available}

The following files are available free of charge.

Alphanumeric strings, 2D root-mean-square deviations, asphericity and shape parameters, normalized atomic and cationic densities, radial distribution function, and volumetric maps of all polymers (PDF).

\section{References}

(1) Collet, H.; Souaid, E.; Cottet, H.; Deratani, A.; Boiteau, L.; Dessalces, G.; Rossi, J.-C.; Commeyras, A.; Pascal, R. An Expeditious Multigram-Scale Synthesis of Lysine Dendrigraft (DGL) Polymers by Aqueous N-Carboxyanhydride Polycondensation. Chem. Eur. J. 2010, 16, 2309-2316.

(2) Kodama, Y.; Nakamura, T.; Kurosaki, T.; Egashira, K.; Mine, T.; Nakagawa, H.; Muro, T.; Kitahara, T.; Higuchi, N.; Sasaki, H. Biodegradable Nanoparticles Composed of Dendrigraft Poly-L-Lysine for Gene Delivery. Eur. J. Pharm. and Biopharm. 2014, $87,472-479$.

(3) Tang, M.; Dong, H.; Li, Y.; Ren, T. Harnessing the PEG-Cleavable Strategy to Balance 
Cytotoxicity, Intracellular Release and the Therapeutic Effect of Dendrigraft Poly- $L$ Lysine for Cancer Gene Therapy. J. Mater. Chem. B 2016, 4, 1284-1295.

(4) Romestand, B.; Rolland, J.-L.; Commeyras, A.; Coussot, G.; Desvignes, I.; Pascal, R.; Vandenabeele-Trambouze, O. Dendrigraft Poly- $L$-Lysine: A Non-Immunogenic Synthetic Carrier for Antibody Production. Biomacromolecules 2010, 11, 1169-1173.

(5) Liu, Y.; An, S.; Li, J.; Kuang, Y.; He, X.; Guo, Y.; Ma, H.; Zhang, Y.; Ji, B.; Jiang, C. Brain-targeted Co-Delivery of Therapeutic Gene and Peptide by Multifunctional Nanoparticles in Alzheimer's Disease Mice. Biomaterials 2016, 80, 33-45.

(6) Yang, Z.-Z.; Li, L.; Wang, L.; Xu, M.-C.; An, S.; Jiang, C.; Gu, J.-K.; Wang, Z.-J. J.; Yu, L.-S.; Zeng, S. siRNA Capsulated Brain-targeted Nanoparticles Specifically Knock Down OATP2B1 in Mice: a Mechanism for Acute Morphine Tolerance Suppression. Sci. Rep. 2016, 6, 33338.

(7) Kodama, Y.; Kuramoto, H.; Mieda, Y.; Muro, T.; Nakagawa, H.; Kurosaki, T.; Sakaguchi, M.; Nakamura, T.; Kitahara, T.; Sasaki, H. Application of Biodegradable Dendrigraft Poly-L-Lysine to a Small Interfering RNA Delivery System. J. Drug Target. 2016, 1-9.

(8) Yao, H.; Wang, K.; Wang, Y.; Wang, S.; Li, J.; Lou, J.; Ye, L.; Yan, X.; Lu, W.; Huang, R. Enhanced Blood-brain Barrier Penetration and Glioma Therapy Mediated by a New Peptide Modified Gene Delivery System. Biomaterials 2015, 37, 345-352.

(9) Han, L.; Guo, Y.; Ma, H.; He, X.; Kuang, Y.; Zhang, N.; Lim, E.; Zhou, W.; Jiang, C. Acid Active Receptor-Specific Peptide Ligand for In Vivo Tumor-Targeted Delivery. Small 2013, 9, 3647-3658.

(10) An, S.; Kuang, Y.; Shen, T.; Li, J.; Ma, H.; Guo, Y.; He, X.; Jiang, C. Brain-targeting Delivery for RNAi Neuroprotection Against Cerebral Ischemia Reperfusion Injury. Biomaterials 2013, 34, 8949-8959. 
(11) Liu, Y.; Guo, Y.; An, S.; Kuang, Y.; He, X.; Ma, H.; Li, J.; Lv, J.; Zhang, N.; Jiang, C. Targeting Caspase-3 as Dual Therapeutic Benefits by RNAi Facilitating Brain-Targeted Nanoparticles in a Rat Model of Parkinson's Disease. PLoS ONE 2013, 8, e62905.

(12) Hofman, J.; Buncek, M.; Haluza, R.; Streinz, L.; Ledvina, M.; Cigler, P. In Vitro Transfection Mediated by Dendrigraft Poly-L-lysines: The Effect of Structure and Molecule Size. Macromol. Biosci. 2013, 13, 167-176.

(13) Liu, Y.; Li, J.; Shao, K.; Huang, R.; Ye, L.; Lou, J.; Jiang, C. A Leptin Derived 30Amino-Acid Peptide Modified Pegylated Poly-L-Lysine Dendrigraft for Brain Targeted Gene Delivery. Biomaterials 2010, 31, 5246-5257.

(14) Huang, R.; Liu, S.; Shao, K.; Han, L.; Ke, W.; Liu, Y.; Li, J.; Huang, S.; Jiang, C. Evaluation and Mechanism Studies of PEGylated Dendrigraft Poly- $L$-Lysines as Novel Gene Delivery Vectors. Nanotechnology 2010, 21, 265101.

(15) Hu, G.; Zhang, H.; Zhang, L.; Ruan, S.; He, Q.; Gao, H. Integrin-mediated Active Tumor Targeting and Tumor Microenvironment Response Dendrimer-gelatin Nanoparticles for Drug Delivery and Tumor Treatment. Int. J. Pharm. 2015, 496, 1057-1068.

(16) Huang, S.; Shao, K.; Liu, Y.; Kuang, Y.; Li, J.; An, S.; Guo, Y.; Ma, H.; Jiang, C. Tumor-Targeting and Microenvironment-Responsive Smart Nanoparticles for Combination Therapy of Antiangiogenesis and Apoptosis. ACS Nano 2013, 7, 2860-2871.

(17) Liu, S.; Guo, Y.; Huang, R.; Li, J.; Huang, S.; Kuang, Y.; Han, L.; Jiang, C. Gene and Doxorubicin Co-Delivery System for Targeting Therapy of Glioma. Biomaterials 2012, 33, 4907-4916.

(18) Sideratou, Z.; Sterioti, N.; Tsiourvas, D.; Tziveleka, L.-A.; Thanassoulas, A.; Nounesis, G.; Paleos, C. M. Arginine End-functionalized Poly-L-Lysine) Dendrigrafts for the Stabilization and Controlled Release of Insulin. J. Colloid Interface Sci. 2010, 351, 433-441. 
(19) Kojima, C.; Fusaoka-Nishioka, E.; Imai, T.; Nakahira, A.; Onodera, H. Dendrigraft Polylysine Coated-poly(glycolic acid) Fibrous Scaffolds for Hippocampal Neurons. J. Biomed. Mater. Res. A 2016, 104, 2744-2750.

(20) Lorion, C.; Faye, C.; Maret, B.; Trimaille, T.; Régnier, T.; Sommer, P.; Debret, R. Biosynthetic Support Based on Dendritic Poly-L-Lysine Improves Human Skin Fibroblasts Attachment. J. Biomater. Sci. Polym. Ed. 2014, 25, 136-149.

(21) Eap, S.; Bécavin, T.; Keller, L.; Kokten, T.; Fioretti, F.; Weickert, J.-L.; Deveaux, E.; Benkirane-Jessel, N.; Kuchler-Bopp, S. Nanofibers Implant Functionalized by Neural Growth Factor as a Strategy to Innervate a Bioengineered Tooth. Adv. Healthc. Mater. 2014, 3, 386-391.

(22) Eap, S.; Ferrand, A.; Schiavi, J.; Keller, L.; Kokten, T.; Fioretti, F.; Mainard, D.; Ladam, G.; Benkirane-Jessel, N. Collagen Implants Equipped with 'Fish Scale'-like Nanoreservoirs of Growth Factors for Bone Regeneration. Nanomedicine 2014, 9, 12531261.

(23) Cadiere, A.; Couturaud, B.; Boismard, J.; Cann, P. L.; Gérard, A.; Mas, A.; Faye, C.; Garrelly, L.; Roig, B. Assessment of Poly-L-Lysine Dendrigrafts for Virus Concentration in Water: Use of MS2 Bacteriophage as Proof of Concept. J. Appl. Microbiol. 2013, $115,290-297$.

(24) Mendoza-Palomares, C.; Ferrand, A.; Facca, S.; Fioretti, F.; Ladam, G.; KuchlerBopp, S.; Regnier, T.; Mainard, D.; Benkirane-Jessel, N. Smart Hybrid Materials Equipped by Nanoreservoirs of Therapeutics. ACS Nano 2012, 6, 483-490.

(25) Fioretti, F.; Mendoza-Palomares, C.; Helms, M.; Alam, D. A.; Richert, L.; Arntz, Y.; Rinckenbach, S.; Garnier, F.; Haikel, Y.; Gangloff, S. C.; Benkirane-Jessel, N. Nanostructured Assemblies for Dental Application. ACS Nano 2010, 4, 3277-3287. 
(26) Han, L.; Liu, M.; Ye, D.; Zhang, N.; Lim, E.; Lu, J.; Jiang, C. Tumor Cell Membranetargeting pH-dependent Electron Donor-Acceptor Fluorescence Systems with Low Background Signals. Biomaterials 2014, 35, 2952-2960.

(27) Li, J.; Huang, S.; Shao, K.; Liu, Y.; An, S.; Kuang, Y.; Guo, Y.; Ma, H.; Wang, X.; Jiang, C. A Choline Derivate-modified Nanoprobe for Glioma Diagnosis using MRI. Sci. Rep. 2013, 3, 1623-1632.

(28) Liu, Y.; Hu, Y.; Guo, Y.; Ma, H.; Li, J.; Jiang, C. Targeted Imaging of Activated Caspase-3 in the Central Nervous System by a Dual Functional Nano-Device. J. Control. Release 2012, 163, 203-210.

(29) Huang, R.; Han, L.; Li, J.; Liu, S.; Shao, K.; Kuang, Y.; Hu, X.; Wang, X.; Lei, H.; Jiang, C. Chlorotoxin-modified Macromolecular Contrast Agent for MRI Tumor Diagnosis. Biomaterials 2011, 32, 5177-5186.

(30) Theodossiou, T. A.; Sideratou, Z.; Tsiourvas, D.; Paleos, C. M. A Novel Mitotropic Oligolysine Nanocarrier: Targeted Delivery of Covalently Bound D-Luciferin to Cell Mitochondria. Mitochondrion 2011, 11, 982-986.

(31) Kojima, C.; Turkbey, B.; Ogawa, M.; Bernardo, M.; Regino, C. A.; Bryant, L. H.; Choyke, P. L.; Kono, K.; Kobayashi, H. Dendrimer-based MRI Contrast Agents: The Effects of PEGylation on Relaxivity and Pharmacokinetics. Nanomedicine: NBM 2011, 7, 1001-1008.

(32) Francoia, J.-P.; Pascal, R.; Vial, L. Monitoring Clinical Levels of Heparin in Human Blood Samples with an Indicator-Displacement Assay. Chem. Commun. 2015, 51, 1953-1956.

(33) Francoia, J.-P.; Vial, L. A KISS (keep it simple, sensor) Array for Glycosaminoglycans. Chem. Commun. 2015, 51, 17544-17547. 
(34) Couturaud, B.; Bondia, A. M.; Faye, C.; Garrelly, L.; Mas, A.; Robin, J. J. Grafting of Poly-L-Lysine Dendrigrafts onto Polypropylene Surface Using Plasma Activation for ATP Immobilization - Nanomaterial for Potential Applications in Biotechnology. $J$. Colloid and Interface Sci. 2013, 408, 242-251.

(35) Koper, G. J. M.; Borkovec, M. Proton Binding by Linear, Branched, and Hyperbranched Polyelectrolytes. Polymer 2010, 51, 5649-5662.

(36) Thurlkill, R. L.; Thurlkill, R. L.; Grimsley, G. R.; Grimsley, G. R.; Scholtz, J. M.; Scholtz, J. M.; Pace, C. N.; Pace, C. N. pK Values of the Ionizable Groups of Proteins. Protein Sci. 2006, 15, 1214-1218.

(37) Pace, C. N.; Grimsley, G. R.; Scholtz, J. M. Protein Ionizable Groups: pK Values and their Contribution to Protein Stability and Solubility. J. Biol. Chem. 2009, 284, $13285-13289$.

(38) DGLs were provided by the Colcom company http://www.colcom.eu (accessed September 2017).

(39) The source codes of the programs are available at https://www.github.com/gmonard/DGL (accessed January 2017).

(40) Macke, T. J.; Case, D. A. In Molecular Modeling of Nucleic Acids; Leontis, N. B., SantaLucia, J., Eds.; American Chemical Society: Washington, DC, 1997; Chapter 24, pp 379-393.

(41) Case, D. A.; Berryman, J. T.; Betz, R. M.; Cerutti, D. S.; Cheatham, T. E.; Darden, T. A.; Duke, R. E.; Giese, T. J.; Gohlke, H.; Goetz, A. W.; Homeyer, N.; Izadi, S.; Janowski, P.; Kaus, J.; Kovalenko, A.; Lee, T. S.; LeGrand, S.; Li, P.; Luchko, T.; Luo, R.; Madej, B.; Merz, K. M.; Monard, G.; Needham, P.; Nguyen, H.; Nguyen, H. T.; Omelyan, I.; Onufriev, A.; Roe, D. R.; Roitberg, A.; Salomon-Ferrer, R.; Simmerling, C. L.; Smith, W.; Swails, J.; Walker, R. C.; Wang, J.; Wolf, R. M.; Wu, X.; 
York, D. M.; Kollman, P. A. AMBER 15: University of California, San Francisco, 2015 .

(42) Nguyen, H.; Maier, J.; Huang, H.; Perrone, V.; Simmerling, C. Folding Simulations for Proteins with Diverse Topologies Are Accessible in Days with a Physics-Based Force Field and Implicit Solvent. J. Am. Chem. Soc 2014, 136, 13959-13962.

(43) Hopkins, C. W.; Grand, S. L.; Walker, R. C.; Roitberg, A. E. Long-Time-Step Molecular Dynamics through Hydrogen Mass Repartitioning. J. Chem. Theory Comput. 2015, 11, $1864-1874$.

(44) Flory, P. J. The Configuration of Real Polymer Chains. J. Chem. Phys. 1949, 17, $303-310$.

(45) Falkovich, S.; Markelov, D.; Neelov, I.; Darinskii, A. Are Structural Properties of Dendrimers Sensitive to the Symmetry of Branching? Computer Simulation of Lysine Dendrimers. Journal of Chemical Physics 2013, 139.

(46) Jin, X.; Leclercq, L.; Sisavath, N.; Cottet, H. Investigating the Influence of Phosphate Ions on Poly(L-lysine ) Conformations by Taylor Dispersion Analysis. Macromolecules 2014, 47, 5320-5327.

(47) The theoretical values of the Flory exponent are 0.33 and 0.59 for hard spheres (i.e. compact spherical molecules) and random coils, respectively.

(48) Hofmann, H.; Soranno, A.; Borgia, A.; Gast, K.; Nettels, D.; Schuler, B. Polymer Scaling Laws of Unfolded and Intrinsically Disordered Proteins Quantified with SingleMolecule Spectroscopy. Proceedings of the National Academy of Sciences 2012, 109, $16155-16160$.

(49) For all the polymers, only the deviations of the residues of the core DGL G1 were 
analyzed during the trajectories. The behavior of these amino acids within a polymer was considered to be representative of the whole macromolecule's behavior.

(50) Rawdon, E. J.; Kern, J. C.; Piatek, M.; Plunkett, P.; Stasiak, A.; Millett, K. C. Effect of Knotting on the Shape of Polymers. Macromolecules 2008, 41, 8281-8287.

(51) The normalized radial atomic (or cationic) density $\rho_{n}(\mathrm{r})$ was obtained by counting the number $\mathrm{N}$ of atoms (or positive charges) whose centers of mass are located within a spherical shell of radius $\mathrm{r}$ and thickness $\Delta \mathrm{r}$. The function $\mathrm{N}(\mathrm{r})$ was then divided by the volume of the shell $\mathrm{V}(\mathrm{r})$ and multiplied by a constant $\Theta: \rho_{n}(\mathrm{r})=\mathrm{N}(\mathrm{r}) \times \Theta / \mathrm{V}(\mathrm{r})=$ $\rho(\mathrm{r}) \times \Theta$, with $\Theta \times \int_{0}^{\infty} \rho(r) d r=1$.

(52) Humphrey, W.; Dalke, A.; Schulten, K. VMD: Visual Molecular Dynamics. J. Mol. Graph. 1996, 14, 33-38.

(53) Goetz, A. W.; Williamson, M. J.; Xu, D.; Poole, D.; Le Grand, S.; Walker, R. C. Routine Microsecond Molecular Dynamics Simulations with AMBER on GPUs. 1. Generalized Born. J. Chem. Theory Comput. 2012, 8, 1542-1555.

(54) Nguyen, H.; Roe, D. R.; Simmerling, C. Improved Generalized Born Solvent Model Parameters for Protein Simulations. J. Chem. Theory Comput. 2013, 9, 2020-2034.

(55) Maier, J. A.; Martinez, C.; Kasavajhala, K.; Wickstrom, L.; Hauser, K. E.; Simmerling, C. ff14SB: Improving The Accuracy of Protein Side Chain and Backbone Parameters from ff99SB. J. Chem. Theory Comput. 2015, 11, 3696-3713.

(56) Hopkins, C. W.; Le Grand, S.; Walker, R. C.; Roitberg, A. E. Long-Time-Step Molecular Dynamics through Hydrogen Mass Repartitioning. J. Chem. Theory Comput. 2015, $11,1864-1874$.

(57) Ryckaert, J.-P.; Ciccotti, G.; Berendsen, H. J. Numerical Integration of the Cartesian 
Equations of Motion of a System with Constraints: Molecular Dynamics of n-alkanes.

J. Comput. Phys. 1977, 23, 327-341. 


\section{Graphical TOC Entry}

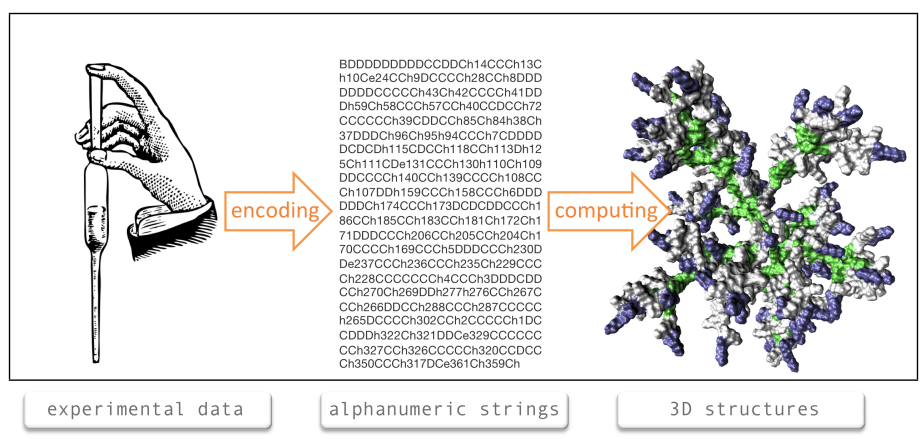

